\title{
Selection of aphasic stroke patients for intensive speech therapy
}

\author{
JULIA A LEGH-SMITH, ROSA DENIS, PAMELA M ENDERBY, DERICK T WADE,* \\ RICHARD LANGTON-HEWER
}

From the Rivermead Rehabilitation Centre* Oxford, and the Stroke Unit, Department of Neurology, Frenchay Hospital, Bristol, UK

SUMMARY Selection criteria for intensive speech therapy and the number of patients fulfilling these were investigated in 441 acute strokes coming from a Health District population during one year. Five patients from a total of 71 referred with speech and/or language difficulties were considered suitable for intensive speech therapy at 4 weeks after stroke. Although such therapy was not actually given, by 26 weeks three of the five had recovered and 14 further patients were considered suitable to receive intensive therapy. The most important selection criteria were Functional Communication Profile score of less than 85 and a clinical judgement that the patient was well enough to take part. It is recommended that decisions as to appropriateness of patients for intensive speech therapy be delayed beyond 4 weeks after stroke.

Stroke is probably the most frequent single cause of impaired communication in adult life and aphasia is the most common reason for adult referrals to speech therapy departments ${ }^{1}$ The effects of therapy in terms of recovery from aphasia are still uncertain.

Conflicting results from previous studies may have arisen from the use of different therapies under different levels of intensity. There is a general lack of clear, repeatable descriptions of therapy regime which has hindered comparison. Also, small samples and a general lack of control groups have meant spontaneous recovery could have been a confounding factor. Most spontaneous recovery has been found to occur up to 3 months post-stroke $e^{2-4}$ in untreated patients, but such recovery may vary with severity of aphasia. ${ }^{5}$ In relation to speech therapy, the use of control nontreatment groups has had differing results. Hagan ${ }^{6}$ and Basso et al $^{7}$ support the effectiveness of speech therapy while Lincoln $e t a l^{8}$ do not.

There are several different types of therapy, the effectiveness of which is still uncertain ${ }^{5910}$ often due to the lack of non-treatment control groups. Howard

Address for reprint requests: Dr R Langton Hewer, Stroke Unit, Department of Neurology, Frenchay Hospital, Bristol BS16 ILE, UK.

Received 11 November 1986 and in revised form 21 April 1987. Accepted 25 April 1987 et $a l^{11}$ comment, however, that "Intriguingly, the studies that report beneficial effects of treatment involved more intensive and prolonged reeducation programmes than the studies that find no effect". The studies referred to include those of Wertz, ${ }^{9}$ Basso et $a l^{7}$ and Vignolo et al. ${ }^{12}$

Intensive therapy has not attracted much attention either from researchers or those providing the service. This may be due to difficulty in finding sufficient therapists. It is necessary, therefore, to make an assessment of management implications before embarking on such a regime. Selection criteria must be established and an estimate obtained of the number of patients for whom this regime could be a realistic possibility. The aims of this study were to: (1) Establish the most appropriate selection criteria for intensive treatment at 4 weeks post-stroke, (2) Ascertain how many aphasic patients fulfill these criteria, (3) Investigate the recovery of potential intensive treatment patients, (4) Establish how many aphasic patients would be suitable for intensive treatment at 26 weeks post-stroke.

\section{Patients}

The sample consisted of 441 acute strokes recorded as occurring between 1 January 1984 and 25 December 1984 (12 mths) in a population of 215,000 people registered with General Practitioners within the Frenchay Health District, Bristol, UK. This register was an extension of that kept between 1 March 1981 and 30 June 1983 and described in 
detail elsewhere. ${ }^{13}$ The diagnosis of stroke was clinical, based on the WHO definition. ${ }^{14}$

\section{Method}

During initial examination by a doctor with special experience of stroke, a clinical judgement was made as to the presence of any language disorder and/or impaired speech and such patients were referred for inclusion in this study. Each referred patient was assessed, at 4 and 26 weeks post-stroke, by the Research Speech Therapist (RD) who had a long experience of post-stroke communication disorders. The Research Speech Therapist took no management decisions relating to treatment of patients. These remained with the responsible doctors and "service" therapists.

Communication was assessed using the Functional Communication Profile (FCP). ${ }^{15}$ This is a standardised test which is simple to administer and is used for quantifying communication behaviours rather than for measuring linguistic deficits. Performance on 45 communication behaviours gives an overall percentage score for five sub-sections: movement, meaning, understanding, reading and other.

Functional ability was assessed using the Barthel Activities of Daily Living Index (ADL) ${ }^{16}$ which has been used in previous studies of stroke patients. ${ }^{13}$ Ability to perform a range of activities of daily living, such as bathing, dressing and transferring gives a total score range of 0-20. A total score of $0-9$ indicates "severe disability"; 10-14 "moderate"; 15-19 "mild"; and, 20 "independent" but not necessarily normal. ${ }^{17}$ Reasoning ability (IQ) was investigated using Raven's Coloured Progressive Matrices. ${ }^{18}$ This is a non-verbal test which has been standardised for elderly people.

Surviving patients in the study were reassessed at 26 weeks post-stroke to establish recovery in communication ability (FCP) after appropriate conventional speech therapy. ADL function was also reassessed.

The factors used initially to exclude patients from the intensive treatment group were an FCP score of 85-100 (not requiring intensive treatment); a Barthel score of 0-9 (severity of disability not conducive to intensive treatment); and, an IQ of less than 60 (marked intellectual deficit). These exclusion criteria are discussed later.

In addition, a clinical rating form was completed by the Research Speech Therapist to provide indications of presence and severity of dysarthria, dyspraxia (as defined by Lesser ${ }^{19}$ ); general physical ability to attend every weekday, all day; and a judgement as to suitability for intensive treatment. This judgement covered illiteracy, blindness, deafness, a foreign native language, transport facilities and each patient's willingness to take part in such a regime.

Intensive therapy was defined as 4 hours per day for 5 days a week. Such treatment requires a patient to be alert, attentive, physically able to sit comfortably, and not to be easily distracted; that is, generally "well enough" to participate. Local conventional therapy varies between one 30 minute session per week to one 30 minute session per weekday.

\section{Results}

A total of $71(16 \%)$ of 441 registered stroke patients within the Frenchay Health District were referred by the study doctor with speech and/or language difficulties. The mean (SD) age of this group was $75.7(9.4)$ years and $30(43 \%)$ were men. Twenty $(28 \%)$ died before 4 weeks; four were unassessable at 4 weeks due to reduced level of consciousness; one was registered after 4 weeks; and one refused to take part. Also, one patient did not use English as her first language and four were blind or deaf. A total of 40 patients were, therefore, assessed by the Research Speech Therapist (RD) at 4 weeks post-stroke and form the basis of this analysis (fig a).

Table 1 shows the distribution of scores for the total group of 40 patients at 4 weeks post-stroke on FCP (mean 65.1, SD 26.9) and Barthel (mean 12.5, SD 6.2). Eighteen patients fell within the selection criteria of FCP less than 85 and Barthel score of more than 9. Two patients were unassessable on FCP due to general behavioural disturbance.

The mean (SD) IQ score for the total group of 40 patients was $89.9(18.8)$ and one patient fell below a Raven's IQ of 60 . Eight $(20 \%)$ patients were unassessable on Raven's due to lack of comprehension of instructions or severe confusion.

Table 2 shows the frequency of dysarthria and dyspraxia at 4 weeks post-stroke. Four patients showed no dysarthria and had FCP scores of more than 90, having recovered by assessment time. Seven patients with dysarthria had FCP scores of more than 85 . The eight $(18 \%)$ patients who had severe dyspraxia had FCP scores of less than 69.

On the basis of the Clinical Rating Form, the Research Speech Therapist considered that only five $(9 \%)$ of the 40 patients were suitable for intensive treatment.

The basis for exclusion from intensive treatment for the study group of $\mathbf{4 0}$ is shown in fig b. Eleven $(30 \%)$ were excluded with FCP scores of 85-100 (language too good). Ten of the remaining 29 were excluded with Barthel scores of 0-6 (physically too disabled). The Barthel cut-off score was lowered from 9 to 6 to allow for one patient scoring 7 who was considered clinically well enough for intensive treatment. None of the remaining 19 patients had an IQ below 60 but six were not "well enough" to take part (four were considered too frail and tired; one was reported medically unstable; one was dementing). The 10 previously excluded on the grounds of a Barthel score less than 6 were also rated as "not well enough" to take part (eight were too frail, ill or tired easily; one was dementing; one in pain).

Thirteen patients could potentially, therefore, take part in an intensive treatment regime but six were unwilling to take part or were uncooperative; one was too tired from other treatments; and two had transport difficulties. The remaining five patients were 
(a) 31 patients excluded before assesment at 4 weeks

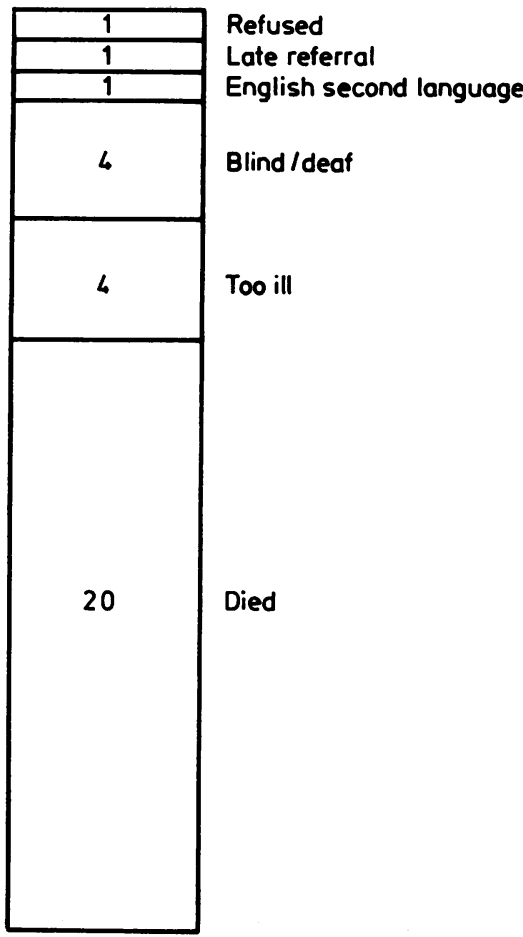

\section{(b) Excluded from study.group at 4 weeks}

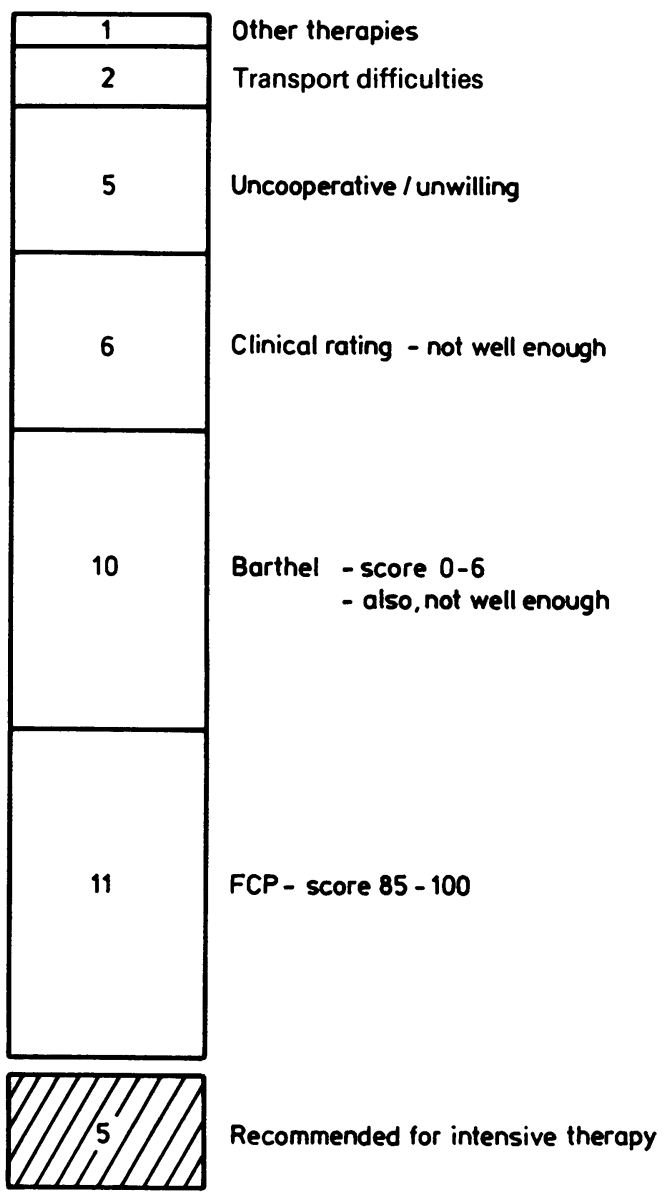

Fig Criteria for exclusion from intensive therapy for 71 patients

those already identified by clinical judgement as being suitable for intensive treatment. Table 3 shows the FCP and Barthel scores for this selected group at 4 and 26 weeks. By 26 weeks three had recovered to within the FCP range of 85-100 but one patient who made a small improvement on FCP deteriorated on Barthel.

Reassessment results at 26 weeks for 19 patients with FCP scores less than 85 at 4 weeks post-stroke are given in table 4 (five patients have been accounted for above; two died during this period; two were too ill at 26 weeks and one was unassessable at 4 weeks). There were no significant improvements in the mean (SD) FCP and Barthel scores for the group. Two patients attained FCP scores of more than 85 and 14 fulfilled both selection criteria (FCP \& Barthel) for intensive treatment, increasing the total to 16 patients at 26 weeks.

\section{Discussion}

This study indicates that only five aphasic stroke patients per year in a Health District population of 215,000 could be included in an intensive treatment regime at 4 weeks post-stroke and three of these recovered with conventional therapy. At 26 weeks post-stroke the number suitable for intensive therapy increased to 16 patients suggesting that decisions as to intensive treatment might be delayed beyond 4 weeks. 
Table 1 Distribution of Barthel and FCP scores for 40 study patients at 4 weeks post stroke

\begin{tabular}{llllll}
\hline \multicolumn{5}{c}{ Barthel $A D L$ score } \\
\cline { 2 - 6 } & $0-9$ & $10-14$ & $15-19$ & 20 & Total \\
\hline FCP score & 2 & & & & \\
$0-19$ & 7 & 6 & 7 & 5 & 25 \\
$20-84$ & 4 & 2 & & 5 & 11 \\
$85-100$ & 2 & & 7 & 10 & 40 \\
Unassessable & 15 & 8 & 7 & 10 \\
Total & & & & & \\
\hline
\end{tabular}

Table 2 Distribution of dysarthria and dyspraxia in 40 study patients

\begin{tabular}{lc}
\hline Dysarthria & \\
0 No dysarthria & $20(50 \%)$ \\
1 Slight dysarthria & $14(35 \%)$ \\
2 Moderate dysarthria & $3(8 \%)$ \\
3 Severe dysarthria & $2(5 \%)$ \\
Unassessable & $1(2 \%)$ \\
Dyspraxia & \\
0 No dyspraxia & $21(52 \%)$ \\
1 Slight dyspraxia & $7(18 \%)$ \\
2 Moderate dyspraxia & $3(8 \%)$ \\
3 Severe dyspraxia & $8(20 \%)$ \\
Unassessable & $1(2 \%)$ \\
\hline
\end{tabular}

Table 3 Outcome in 5 patients suitable for intensive therapy at 4 weeks

\begin{tabular}{llllll}
\hline & \multicolumn{2}{l}{ Barthel Score } & & \multicolumn{2}{l}{$F C P$} \\
\cline { 2 - 3 } \cline { 5 - 6 } Patient & $4 W k$ & $26 W k$ & & $4 W k$ & $26 W k$ \\
\hline 1 & 17 & 19 & 66 & 83 \\
2 & 19 & 20 & & 78 & 92 \\
3 & 20 & 20 & & 67 & 85 \\
4 & 7 & 18 & & 76 & 97 \\
5 & 20 & 16 & & 60 & 65 \\
\hline
\end{tabular}

Table 4 Outcome in 19 patients not recommended for intensive treatment at 4 weeks

\begin{tabular}{lrr}
\hline & 4 Weeks & 26 Weeks \\
\hline FCP Score & & \\
$0-19$ & $3(16 \%)$ & $2(11 \%)$ \\
$20-84$ & $16(84 \%)$ & $15(78 \%)$ \\
$85-100$ & 0 & $2(11 \%)$ \\
Mean (SD) & $48.9(22 \cdot 2)$ & $60.4(24 \cdot 5)$ \\
Barthel ADL Score & & \\
$0-6$ & $5(26 \%)$ & $3(16 \%)$ \\
$7-14$ & $7(37 \%)$ & $5(26 \%)$ \\
$15-19$ & $5(26 \%)$ & $7(37 \%)$ \\
20 & $2(11 \%)$ & $4(21 \%)$ \\
Mean (SD) & $11 \cdot 6(5 \cdot 9)$ & $14.3(5 \cdot 4)$ \\
\hline
\end{tabular}

The principal selection criteria were FCP score (0-84) and general well being.

We consider that the population studied is representative of the majority of stroke patients. No other patients from the Frenchay Health District with stroke induced dysphasia were referred to the Speech Therapy Department from other sources during this time. Also, patients registered for speech/language problems showed a wide range of ability on the FCP as would be expected.

At 4 weeks post-stroke the most relevant criteria were FCP score and clinical judgement of general well being. Criteria based on functional ability (Barthel ADL) and reasoning ability (Raven's IQ) did not identify all those excluded nor did they identify any additional patients.

Reassessment of survivors at 26 weeks post-stroke suggests that it may be appropriate to delay decisions as to suitability for intensive therapy. Of the five patients selected at 4 weeks, three recovered to such an extent that intensive treatment (in hindsight) would not have been necessary (an additional patient attained an FCP score of 83). The one patient who made only a small recovery on FCP showed a functional deterioration suggesting he may not have been able to sustain such a regime. Reassessment at 26 weeks post-stroke for those previously excluded suggested a further 14 patients might be suitable. They fufilled FCP and Barthel selection criteria but would need to be reassessed for general well being and willingness to participate.

Alternatively, redefining "intensive treatment" by, for example, offering 3 days per week instead of 5 , might be less demanding and allow inclusion of more patients at 4 weeks. In terms of effectiveness, however, this study suggests that those more likely to be included at this time will often recover without intensive therapy.

Debate continues as to the most appropriate point in time post-stroke for effective therapy, depending largely on adaptability of the brain following damage. However, whatever the theoretical argument, this study suggests that the majority of aphasic patients needing treatment will not be fit enough to participate in an intensive treatment regime during the early, possibly crucial, period post-stroke. At the same time, concern that such programmes would overload speech therapy resources may be dispelled and intensive speech therapy may be considered a more practical possibility than previously envisaged for those few patients who are considered suitable to receive it.

In conclusion, it is suggested that if research into the effectiveness of an intensive treatment regime is to be undertaken with patients selected within the first 4 weeks post-stroke a very large base population would 
be needed and the study would be a lengthy one, possibly involving several centres. It seems unlikely that any large number of patients suitable for intensive therapy would arise within the average Health District.

We are grateful to the Chest, Heart and Stroke Association for funding this and related studies and thank the patients for their cooperation.

\section{References}

1 Enderby P, Philipp R. Speech and language handicap: towards knowing the size of the problem. $\mathrm{Br} J \mathrm{Dis}$ Com 1986;21:151-65.

2 Sarno MT, Levita E. Natural course of recovery in aphasia. Arch Phys Med Rehabil 1971;52:175-8.

3 Darley FL. Aphasia. WB Saunders, Philadelphia. 1982.

4 Lendram W, Lincoln NB. Spontaneous recovery of language in patients with aphasia between 4 and 34 weeks after stroke. J Neurol Neurosurg Psychiatry 1985;48:743-8.

5 Smith A. Diagnosis, Intelligence and Rehabilitation of Chronic Aphasics. Ann Arbor, University of Michigan. Department of Physical Medicine and Rehabilitation. 1972.

6 Hagan C. Communicative abilities in hemiplegia: effect of speech therapy. Arch Phys Med Rehabil 1973;54: 454-63.

7 Basso A, Capitani E, Vignolo L. Influence of rehabilitation on language skills in aphasic patients: a controlled study. Arch Neurol 1979;36:190-6.

8 Lincoln N, McGuirk E, Mulley GP, Jones AC, Mitchell JKA. Effectiveness of speech therapy for aphasic stroke patients. A randomised controlled trial. Lancet 1984;i:1197-200.

9 Wertz RT, Collins MJ, Brookshire RH. The Veterans Administration Co-operative study on aphasia: a comparison of individual and group treatment. $J$ Speech Hearing Res 1978;24:580-94.

10 Lincoln BL, Pickersgill MJ. The effectiveness of programmed instruction with operant training in the language rehabilitation of severely aphasic patients. Behav Psych Therapy 1984;12:237-48.

11 Howard D, Patterson K, Franklin S, Orchard-Lisle V, Morton J. Treatment of word retrieval deficits in aphasia. Brain 1985;108:817-29.

12 Vignolo LA. Evolution of aphasia and language rehabilitation: a retrospective exploratory study. Cortex 1964;1:344-67.

13 Wade DT, Langton-Hewer R, Skilbeck CE, Bainton D, Burns-Cox C. Controlled trial of a home-care service for acute stroke patients. Lancet 1985;i:323-6.

14 Aho K, Harmsen P, Hatano S, Marquardsen J, Smirnov VI, Strasser T. Cerebrovascular disease in the community: results of a WHO collaborative study. Bull WHO 1980;58:113-80.

15 Sarno TM. The Functional Communication Profile. Manual of Directions. New York University Medical Centre: Rehabilitation Monograph 1969;69.

16 Granger CV, Davis LS, Peters NC, Sherwood CC. Stroke rehabilitation: analysis of repeated Barthel index measures. Arch Phys Med Rehab 1979;60:14-7.

17 Wade DT, Langton-Hewer R. Functional ability after stroke: reassessment, natural history and prognosis. $J$ Neurol Neurosurg Psychiatry 1987;50:177-182.

18 Raven JC. Guide to Using the Coloured Progressive Matrices. London: Lewis \& Co. 1965.

19 Lesser $R$, Watt $M$. Untrained community help in the rehabilitation of stroke sufferers with language disorder. Br Med J 1978;2:1045-8. 